\title{
Calidad de vida relacionada con la salud, depresión y progresión de enfermedad en sobrevivientes de cáncer de mama venezolanas
}

\author{
Health-related quality of life, depression \\ and disease progression in Venezuelan breast cancer survivors
}

\author{
José Javier Altuve Burgos y Andrés Antonio Parra \\ Universidad Central de Venezuela ${ }^{1}$ \\ Autor para correspondencia: José Javier Altuve Burgos, a.altuve.07@gmail.com.
}

\begin{abstract}
RESUMEN
El objetivo de este estudio fue determinar la relación entre la calidad de vida relacionada con la salud (CVRS), la depresión y la progresión de enfermedad en pacientes con Cáncer de mama venezolanas que llevaban corto tiempo de sobrevivencia. Participaron 203 mujeres, de edades entre 24 y 75 años. Se emplearon la subescala de depresión de la Escala de Ansiedad y Depresión Hospitalaria (HADS), el Cuestionario de Salud General Reducido (GHQ-R), el Cuestionario de Salud Global SF-12 y, a través de la Tomografía Computarizada (TC) de las participantes, se evaluó la presencia o ausencia de progresión de enfermedad. El 7.4\% de las pacientes reportaron nivel elevado de depresión, los niveles de CVRS fueron conservados y al $67 \%$ de la muestra no se le detectó progresión de enfermedad en sus TC. No se encontró asociación entre la depresión, la CVRS y la progresión de enfermedad. La depresión correlacionó inversa y significativamente con la CVRS y la misma dirección se reportó cuando se examinó esta relación con respecto a la presencia o ausencia de progresión de enfermedad detectada en las pacientes. Se precisó que la fuerza en la relación entre la depresión y la CVRS fue mayor en las pacientes que se les identificó progresión de enfermedad.
\end{abstract}

Palabras clave: Depresión; Calidad de vida relacionada con la salud; Progresión de enfermedad; Cáncer de mama; Psicooncología.

\begin{abstract}
The objective of this study was to determine the relationship between health-related quality of life (HRQOL), depression, and disease progression in Venezuelan breast cancer patients having a short survival time. A total of 203 women, aged between 24 and 75 years, participated. Data collection included the depression subscale of the Hospital Anxiety and Depression Scale (HADS), the Reduced General Health Questionnaire (GHQ-R), the SF-12 Global Health Questionnaire. Computed Tomography (CT) of the Participants assessed for the presence or absence of disease progression. Results revealed that $7.4 \%$ of the patients reported a high level of depression, preserved levels of CVRS, and $67 \%$ of the sample had no disease progression detected through CT scans. No association resulted between depression, HRQoL, and disease progression. Depression inversely and significantly correlated with HRQOL. The same direction occurred with the relationship regarding the presence or absence of disease progression. Thus conclusions point in the direction that the strength in the
\end{abstract}

\footnotetext{
${ }^{1}$ Escuela de Psicología, Facultad de Humanidades y Educación, 1053 Caracas, D.C., Caracas, Venezuela, correos electrónicos: a.altuve.07@ gmail.com y oncologoparra@gmail.com.
} 
relationship between depression and HRQOL was more significant in the patients who showed disease progression.

Key words: Depression; Health-related Quality of Life; Disease progression; Breast cancer; Psycho-Oncology.

Recibido: 06/08/2020

Aceptado: 11/11/2020

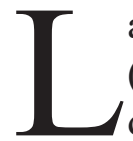

a calidad de vida relacionada con la salud (CVRS) es un constructo comúnmente usado en el contexto de las enfermedades crónicas que se refiere a un fenómeno subjetivo en el que los individuos perciben el efecto negativo o positivo que tiene una enfermedad y sus tratamientos sobre su bienestar en las esferas física, cognitiva, emocional y social (Rutherford, 2018). El cáncer, a su vez, es una enfermedad crónica que altera todas esas áreas, tanto en el paciente, como en su familia y en sus redes de apoyo (Die Trill, 2003; Holland et al., 2015). De todas las neoplasias existentes, el cáncer de mama es uno de los que más se diagnostica en las mujeres y representa $16 \%$ de todos los cánceres femeninos. En Venezuela se registran 2,067 defunciones por este tipo de cáncer y se a ha estimado que las pacientes pueden llegar a tener una sobrevida mayor a dos años en el pais (Altuve, 2014; Rodríguez et al., 2014).

Ante un diagnóstico de cáncer de mama, es de esperarse que las mujeres manifiesten respuestas emocionales transitorias acompañadas con síntomas ansiosos y depresivos que, al intensificarse, pueden incidir en su calidad de vida y salud (Altuve, 2017; Beatty y Kissane, 2017; Cardoso, Graca, Klut, Trancas y Papolia, 2015; Cruzado, 2014: Die Trill, 2003; Holland et al., 2015; Jaras y Gutiérrez, 2016; Smith, 2015). Aunque la ansiedad es más prevalente en los pacientes oncológicos, la depresión ha recibido una importante atención dada su vinculación con la incidencia y letalidad del cáncer (Bernal y Muñoz., 2016; Diz, Garza, Olivas, Montes y Fernández, 2019; Lutgendorf y Andersen, 2015; Massie, 2004).
La depresión es un síndrome clínico clasificado como un trastorno mental del estado del ánimo que se caracteriza por una sensación de abatimiento emocional, incapacidad para percibir alegría o placer y presencia de pensamientos negativos (Beck, 1987). En la depresión pueden gestarse sentimientos profundos de vacío, tristeza, culpa, inferioridad e inutilidad; dificultades para la atención, concentración, memoria, toma de decisiones y juicios; sensación de enlentecimiento, agitación psicomotriz, reducción de la energía y de la voluntad, aislamiento, presencia de pensamientos o conductas autodestructivas y suicidas; síntomas fisiológicos tales como fatiga y alteración del sueño, de la ingesta de alimentos y del peso, al igual que una diminución de la libido o del placer. Todos estos síntomas han mostrado intensificarse durante al menos dos semanas seguidas para lograr precisar la presencia de un trastorno depresivo mayor (Bernal y Muñoz, 2016; Diz et al., 2019).

La prevalencia de la sintomatología depresiva en los pacientes oncológicos oscila entre $1 \mathrm{y}$ $50 \%$, y la depresión mayor en $12.5 \%$ (Caruso et al., 2016; Diz et al., 2019; Holland et al., 2015; Zabora, Brintzenhofeszoc, Curbow, Hooker y Piantadosi, 2001). En cuanto al cáncer de mama, se ha encontrado una prevalencia de depresión de 32 a $53 \%$ en la población general (Pilevarzadeh et al., 2019; Zabora et al., 2001) y de 25 a 52\% en pacientes latinas hispanoparlantes (Ashing-Giwaet, Rosales, Lai y Weitzel, 2012; Holden, Ramirez y Gallion, 2014; Lee et al., 2017; Linden, Vodermaier, MacKenzie y Greig, 2012). Otros estudios hechos en Latinoamérica, como el de Valderrama y Sánchez (2018), registraron una prevalencia de depresión clínica en pacientes con cáncer de mama de $25 \%$, mientras que Parra (2020), en Venezuela, reportó $6.3 \%$ en pacientes que se encontraban en la fase de tratamiento y libre de enfermedad.

Hay bases para afirmar que las pacientes con cáncer de mama que reportan elevado malestar psicológico tienen una reducción significativa de su CVRS, reflejada en una disminución de su desempeño físico, funcionamiento social y de sus roles en la vida diaria, al igual que dificultades cogni- 
tivas, cambios en su imagen corporal y reducción del deseo sexual, sin que se afecte el funcionamiento familiar (Beatty y Kissane, 2017; Park et al., 2018; Reich, Lesur y Perdrizet-Chevallier, 2008). Recientemente, Fernández et al. (2020) evaluaron la CVRS a través de los Cuestionarios de Salud Global de Goldberg y el SF-36 en una muestra de 1,017 mujeres con cáncer de mama españolas, encontrando que las que reportaban más distrés exhibían también una baja CVRS. Pero existe evidencia específica de que la depresión deteriora directamente la CVRS de las pacientes con cáncer de mama (De Ligt et al., 2019; Franco, Zuluaga, Vinaccia, Raleigh y Martínez, 2019; Grabsch et al., 2006; Høyer et al., 2011; Reich et al., 2008; Schou, Ekeberg, Sandvik, Hjermstad y Ruland, 2005; Yfantis et al., 2018).

Se ha reportado que la incidencia de la depresión es independiente del estadio o la gravedad de la enfermedad, el tipo de tratamiento y el nivel de discapacidad física percibida. Su mayor prevalencia suele ocurrir en el primer año tras el diagnóstico, con una tendencia a decrecer. Sin embargo, ante la recurrencia o en la etapa paliativa suele aumentar, junto con la aparición de síntomas fisiológicos (Bortolato et al., 2017), pues el cáncer y la depresión suelen coexistir (Spiegel y Giese-Davis, 2003). La depresión comórbida se ha asociado con la ocurrencia de hospitalizaciones frecuentes y prolongadas, con una baja adherencia al tratamiento, un peor pronóstico del cáncer y una mayor mortalidad precedida por la progresión de la enfermedad (Bernal y Muñoz, 2016; Diz et al., 2019; Lutgendorf y Andersen, 2015). La progresión de la enfermedad ocurre cuando un tumor cancerígeno comienza a aumentar de tamaño en el mismo sitio en que se desarrolló inicialmente y se disemina hacia otras partes del cuerpo, proceso conocido comúnmente como metástasis (Lutgendorf y Sood, 2011).

Existe evidencia de que la depresión incide directa e indirectamente sobre la progresión del cáncer (Bortolato et al., 2017; Costanzo, Sood y Lutgendorf, 2011; Lutgendorf y Andersen, 2015; Reiche, Nunes y Morimoto, 2004; Young y Singh, 2018). Específicamente, se ha descubierto que la depresión puede influir en la regulación del eje hipotalámico-pituitario-adrenal y del sistema inmune, así como en los procesos inflamatorios y angiogenéticos que son importantes en el control y desarrollo de la progresión del cáncer (Bortolato et al., 2017; Lutgendorf y Andersen, 2015; Pinquart y Duberstein, 2010; Young y Singh, 2018). Spiegel y Giese-Davis (2003) han apuntado que la progresión de la enfermedad no solo se debe al efecto fisiopatológico que la depresión causa en la función inmunológica, sino también en los efectos que tiene en la disminución de la adherencia a los tratamientos oncológicos y las conductas de prevención y control, los que producen que el cáncer se agrave y su pronóstico se vea afectado. No obstante, Lutgendorf y Andersen (2015) señalan que los estudios sobre la vinculación entre la depresión y la progresión oncológica aún no han sido concluyentes. Un estudio de Mansano y Ceolim (2017), cuyo objetivo era establecer la relación causal entre pronóstico clínico desfavorable y la duración del sueño en una muestra de 114 pacientes con cáncer de mama colombianas, encontró depresión severa en $54 \%$ de las pacientes que progresaron y en las que no lo hicieron. Además, hallaron que la depresión no resultó ser un predictor de la progresión clínica desfavorable en su muestra de pacientes.

En sintesis, se ha reportado evidencia de que la depresión deteriora la CVRS de los pacientes oncológicos, pero su asociación con la progresión del cáncer todavía no es concluyente, lo que subraya la importancia de seguir efectuando más estudios en esta área, especialmente en contextos latinoamericanos. Dado que el cáncer de mama es una de las patologías oncológicas diagnosticadas frecuentemente en Venezuela, y que los estudios en el país han sido escasos -según la revisión bibliográfica realizada sobre las reacciones emocionales y su influencia en los procesos de salud-enfermedad en pacientes con este tipo de neoplasia -, la presente investigación, de corte correlacional con diseño no experimental (Hernández, Fernández y Baptista, 2012; Kerlinger, 2002), tuvo como objetivo determinar las relaciones entre la CVRS, la depresión y la progresión de la enfermedad en un muestra de pacientes venezolanas con cáncer de mama. 


\section{MÉTODO}

\section{Participantes}

En el estudio participaron 203 pacientes con diagnóstico de cáncer de mama, confirmado histológicamente, con edades de 24 a 75 años $(M=56$, D.E. $=12.17)$ que asistían al Servicio Oncológico Hospitalario (Antiguo Hospital "Padre Machado") de la cuidad de Caracas, Venezuela, que llevaban un corto tiempo de sobrevivencia ( $<5$ años). El muestreo realizado fue de tipo no probabilístico (Kerlinger y Lee, 2002) en virtud de que la selección se hizo siguiendo las características que interesaban a los investigadores. La recolección de los datos tuvo una duración de ocho meses entre los años 2019 y 2020. Los criterios de inclusión y exclusión que se siguieron fueron que todas las pacientes estuvieran en la fase libre de enfermedad; que ya hubiesen finalizado los tratamientos de quimioterapia y radioterapia; que todas ellas expresaran participar de forma voluntaria en el estudio y refirieran estar en buen estado de ánimo; que no reportaran dificultades físicas, tales como dolor, fatiga e insomnio ni dificultades para atender y comprender, y que supieran leer y escribir. No se pidió la participación de aquellas que no cumplieran los criterios antes mencionados.

\section{Instrumentos}

Se hizo un estudio piloto en el que se revisaron las escalas que se describen a continuación, a fin de adaptarlas a la población objetivo. Esta revisión contó con la participación de dos especialistas en psicooncología. Una vez revisados los instrumentos, se llevó a cabo un estudio piloto con 100 pacientes venezolanas con cáncer de mama, con edades de 25 a 70 años, que estaban en las fases de tratamiento y libres de enfermedad.

\section{Cuestionario de Salud General de Goldberg (GHQ)} (Goldberg y Hillier. 1979).

Se empleó la versión colombiana propuesta por Villa, Zuluaga y Restrepo (2013), denominada por estos autores como Cuestionario de Salud General Reducido (GHQ-R). La escala fue validada en pacientes con diferentes diagnósticos médicos. Los ítems se pueden responder en dos escalas; una escala tipo Likert de cuatro puntos (0-1-2-3), con las opciones de respuesta: "Más que antes", "Igual que antes", "Menos que antes" y "No, nunca", o bien en un puntaje binario (0-0-1-1). Puntajes mayores en la escala reflejan una mejor salud general en los pacientes. La escala contiene once reactivos agrupados en dos dimensiones: Bienestar y Malestar psicológico, que reportaron una consistencia interna adecuada $(\alpha=0.84)$. El índice de confiabilidad obtenido en el estudio piloto antes mencionado fue de 0.73 .

Cuestionario de Salud SF-12 (versión 2)

(Ware, Kosinski y Keller, 1996).

Se empleó la versión adaptada en Venezuela por Hernández, Salas y Altuve (2020). Este cuestionario evalúa la CVRS y consta de doce ítems integrados en ocho dimensiones: función física, rol físico, dolor corporal, salud general, vitalidad, función social, rol emocional y salud mental. Tales dimensiones se agrupan a su vez en dos componentes sumatorios: Salud física y Salud mental. El número de opciones de respuesta oscila entre tres y seis, según el ítem. Puntajes mayores indican una alta CVRS. Los índices de confiabilidad reportados para la dimensión de salud física, mental y global fueron de $0.72,0.73$ y 0.83 , respectivamente. En el estudio piloto mencionado, los índices de confiabilidad obtenidos fueron de 0.74 para Salud física, de 0.76 para Salud mental y de 0.79 para Salud global.

Escala de Ansiedad y Depresión Hospitalaria (HADS) (Zigmond y Snaith, 1983).

Esta escala, adaptada por Rico, Restrepo y Molina (2005), está conformada por 14 reactivos distribuidos en dos dimensiones: Ansiedad y Depresión, cada una con siete ítems. En esta escala se excluyen los síntomas somáticos, lo que evita equívocos al momento de valorar a pacientes con alguna enfermedad. Para efectos de este estudio, se utilizaron solamente los puntajes de la subescala de Depresión, que se centra exclusivamente en la anhedonia, síntoma central de esta afección. Dicha subescala, según datos reportados por Rico et 
al. (2005), muestra un índice de confiabilidad de 0.76 . El coeficiente de confiabilidad obtenido en el estudio piloto fue de 0.72 .

\section{Tomografía computarizada}

La progresión de la enfermedad se evaluó mediante el examen de imágenes de tomografía computarizada (TC) con doble contraste del abdomen, pelvis y tórax, que las pacientes de la muestra presentaron en un DVD, junto con el informe del radiólogo responsable. La TC es un estudio de imágenes en el que se puede visualizar el cuerpo (órganos, huesos y tejido blando) con mayor claridad mediante un corte o una sección trasversal. Es útil para diagnosticar neoplasias o tumores de acuerdo a su forma, tamaño y ubicación, e incluso los vasos sanguíneos a través de los cuales se alimentan estas lesiones. Este estudio permite precisar cómo un tumor responde al tratamiento, o si ha recurrido o progresado durante o tras su aplicación (Holland et al., 2015). La presencia o la ausencia de progresión de enfermedad se confirmó con la observación de imágenes que sugirieran posibles lesiones tumorales en otros órganos distintos a la localización del tumor primario que, en el caso de las pacientes de la muestra, era el seno.

\section{Procedimiento}

La recolección de los datos para el estudio piloto y el análisis de estos se llevaron a cabo a lo largo de dos meses, y ocho para la investigación propiamente dicha. A cada paciente que asistía a la consulta de oncología médica para el seguimiento médico de la enfermedad se le solicitaba su colaboración para el llenado de las escalas, siempre que cumplieran los criterios de inclusión. Una vez que se corroborara lo anterior, se les hacía entrega del formato de consentimiento informado, en el que se exponían los lineamientos éticos relativos a la participación voluntaria, el uso y los fines de los resultados, el anonimato y la confidencialidad de los mismos.

Antes de llevarse a cabo la consulta médica todas las pacientes completaron las escalas de medida; luego de que la consulta terminaba se tomaba la información del número de la historia clínica de cada una para tener acceso al informe de la tomografía computarizada y el DVD que contenía las imágenes, mismas que fueron revisadas a través del programa RadiAnt, un software que permite su examen. Uno de los investigadores, médico oncólogo, revisó las imágenes de las tomografías, leyó las conclusiones de los radiólogos que firmaban el informe de cada estudio y validó toda la información considerando la opinión de otros dos médicos, también especialistas en medicina oncológica y adjuntos al Servicio Oncológico Hospitalario, con experiencia de alrededor de 10 años. Lo anterior se realizó con el objetivo de que precisaran la presencia o ausencia de progresión de la enfermedad en las pacientes. Una vez recolectados todos los datos, los investigadores procedieron a registrarlos en una base de datos de Excel Office, que luego se exportó al programa del paquete estadístico SPSS, versión 22, para los análisis correspondientes.

\section{Análisis de datos}

Los datos se procesaron por medio del paquete estadístico de SPSS, versión 24. Para el análisis descriptivo se emplearon las frecuencias y las medidas de tendencia central y de variabilidad. Obedeciendo al nivel de medida de cada una de las variables, en el análisis de las correlaciones se emplearon la prueba de chi cuadrada y la prueba de momento de Pearson. Para el análisis de la consistencia interna de las escalas empleadas en este estudio se empleo el coeficiente alfa de Cronbach.

\section{RESULTADOS}

En la Tabla 1 se refleja la información sociodemográfica y de salud de las pacientes sobrevivientes de cáncer de mama. Las pacientes en promedio tenían 56 años y llevaban entre 3 a 4 años con el diagnóstico. Se reporta además el tiempo transcurrido, en promedio de un mes, entre la realización de la TC y la consulta médica, en la que también fueron aplicados los instrumentos de medición. El tiempo promedio en que las pacientes estaban en la condición libre de enfermedad fue de 3 años. 
Tabla 1. Datos sociodemográficos y de salud de la muestra.

\begin{tabular}{|c|c|}
\hline CARACTERÍSTICA & M (D.T.) / \% (F) \\
\hline Edad & $56.8 ;(12.1)$ \\
\hline Tiempo con la enfermedad & $3.48 ;(3.37)$ \\
\hline Tiempo entre la realización de la TC y la consulta médica & $1.5(.89)$ \\
\hline Tiempo en la fase libre de enfermedad & $3.0(2.86)$ \\
\hline \multicolumn{2}{|l|}{ Comorbilidad de otras enfermedades } \\
\hline Sí & $29 \%(.58)$ \\
\hline No & $71 \%(145)$ \\
\hline \multicolumn{2}{|l|}{ Antecedentes psiquiátricos } \\
\hline No & $83 \%(169)$ \\
\hline Sí & $17 \%(34)$ \\
\hline Tx. Adaptativo mixto & $68 \%(23)$ \\
\hline Tx. Depresivo mayor & $32 \%(11)$ \\
\hline \multicolumn{2}{|l|}{ Con medicación actual } \\
\hline Sí & $41 \%(14)$ \\
\hline No & $59 \%(20)$ \\
\hline \multicolumn{2}{|l|}{ Tratamiento recibido } \\
\hline Quimioterapia (QT) & $71 \%(143)$ \\
\hline Radioterapia (RT) & $13 \%(27)$ \\
\hline $\mathrm{QT}+\mathrm{RT}$ & $16 \%(33)$ \\
\hline \multicolumn{2}{|l|}{ Manejo adecuado de efectos secundarios } \\
\hline Sí & $83 \%(168)$ \\
\hline No & $17 \%(35)$ \\
\hline \multicolumn{2}{|l|}{ Estrato socioeconómico } \\
\hline Bajo & $53 \%(108)$ \\
\hline Medio & $46 \%(93)$ \\
\hline Alto & $1 \%(2)$ \\
\hline \multicolumn{2}{|l|}{ Estado civil } \\
\hline Casadas & $33 \%(66)$ \\
\hline Solteras & $48 \%(98)$ \\
\hline Divorciadas & $6 \%(13)$ \\
\hline Viudas & $13 \%(26)$ \\
\hline \multicolumn{2}{|l|}{ Madre } \\
\hline Sí & $82 \%(167)$ \\
\hline No & $18 \%(36)$ \\
\hline \multicolumn{2}{|l|}{ Actividad laboral } \\
\hline Sí & $77 \%(156)$ \\
\hline No & $23 \%(47)$ \\
\hline \multicolumn{2}{|l|}{ Asistencia al Servicio de Psicología } \\
\hline Sí & $14 \%(29)$ \\
\hline No & $86 \%(174)$ \\
\hline \multicolumn{2}{|l|}{ Apoyo social y familiar } \\
\hline Sí & $90 \%(183)$ \\
\hline No & $10 \%(20)$ \\
\hline
\end{tabular}


Muchas de las pacientes no manifestaron comorbilidad con otras afecciones ni antecedentes psiquiátricos. En las 34 pacientes que reportaron tales antecedentes, su diagnóstico fue trastorno adaptativo mixto o trastorno depresivo mayor, y en su mayoría no tenían medicación actual. Varias de estas pacientes fueron tratadas con quimioterapia y reportaron haber manejado adecuadamente los efectos secundarios. Pertenecían al estrato socioeconómico bajo, con estado civil de solteras y casa- das, madres que se hallaban activas laboralmente, percibían apoyo social y familiar y no habían asistido al Servicio de Psicología.

Después, se obtuvieron las frecuencias de pacientes que en su tomografía revelaron progresión de la enfermedad. Se puede apreciar que, de la muestra total de pacientes con cáncer de mama, en 136 de ellas (67\%) no se identificó progresión, a diferencia del resto (67 pacientes, esto es, 33\%), en quienes el resultado fue positivo.

Tabla 2. Estadísticos descriptivos para depresión y CVRS.

\begin{tabular}{|l|r|c|c|c|}
\hline \multicolumn{1}{|c|}{ Instrumento } & Mín. & Max. & M & D.E \\
\hline Depresión (HADS) & 0 & 16 & 3.82 & 3.23 \\
\hline Salud General (GHQ-R) & 8 & 31 & 21.3 & 5.43 \\
\hline Salud Física (SF-12) & 6 & 20 & 18.4 & 3.21 \\
\hline Salud Mental (SF-12) & 6 & 27 & 15.3 & 4.25 \\
\hline Salud General (SF-12) & 12 & 47 & 32.3 & 4.20 \\
\hline
\end{tabular}

Tal como se aprecia en la tabla anterior, las pacientes con cáncer de mama obtuvieron un puntaje medio de 3.8, el cual indica que, en promedio, los niveles de depresión fueron bajos. Se encontró que 154 pacientes $(75 \%)$ reportaron depresión baja, $34(16 \%)$ nivel moderado y 15 (7.4\%) nivel grave. La salud general de las pacientes obtuvo una media de 21.3, que denota niveles de salud conservados en la muestra según el GHQ-R. En los indicadores reflejados en el cuestionario SF-12 se halló que las pacientes obtuvieron una media de 18.4 en su salud física y de 15.3 en la mental, los cuales indican, para efectos de esta medida, que ambas dimensiones se encontraban conservadas. La misma tendencia se observó en el promedio de la salud general de SF-12, con una media de 32.3, revelando una adecuada CVRS. Los resultados de las mediciones de la salud fueron similares y consistentes.
Tabla 3. Relación entre la depresión, la CVRS y la progresión de enfermedad.

\begin{tabular}{|l|c|c|}
\cline { 2 - 3 } \multicolumn{1}{c|}{} & \multicolumn{2}{c|}{ Progresión } \\
\cline { 2 - 3 } \multicolumn{1}{c|}{} & $\boldsymbol{X}^{\mathbf{2}}$ & $\boldsymbol{p} \leq \mathbf{0 . 0 5}$ \\
\hline Depresión & 3.722 & .156 \\
\hline Salud (GHQ-R) & 1.214 & .545 \\
\hline Salud (SF-12) & 2.321 & .462 \\
\hline
\end{tabular}

Como se observa en la tabla, los análisis de correlación mediante la prueba de chi cuadrada $\left(X^{2}\right)$ indica que, bajo 95\% de confianza, no hay asociación entre la depresión y la progresión de la enfermedad $\left(X^{2}=3.722\right.$, gl. $\left.=2, p=.156<0.05\right)$. De igual manera, se aprecia en los indicadores de salud evaluados por el GHQ-R $\left(X^{2}=1.214, \mathrm{gl}=2\right.$, $p=.545<0.05)$ y el SF-12 $\left(X^{2}=2.321, \mathrm{gl}=2\right.$, $p=.462<0.05)$, lo que implica que no existe asociación entre la CVRS y la progresión de la enfermedad.

Tabla 4. Correlaciones entre la depresión y CVRS.

\begin{tabular}{|c|c|c|c|c|}
\hline & & & $\begin{array}{c}\text { Salud General } \\
\text { (GHQ-R) }\end{array}$ & $\begin{array}{c}\text { Salud General } \\
\text { (SF-12) }\end{array}$ \\
\hline \multirow{2}{*}{ Progresión } & \multirow{2}{*}{ Depresión } & Correlación de Pearson & $-.487 * *$ & $-.513 * *$ \\
\hline & & Sig. (bilateral) & .000 & .000 \\
\hline \multirow{2}{*}{ No } & \multirow{2}{*}{ Depresión } & Correlación de Pearson & $-.473 * *$ & $-.4 .86 * *$ \\
\hline & & Sig. (bilateral) & .000 & .000 \\
\hline \multirow{2}{*}{ Sí } & \multirow{2}{*}{ Depresión } & Correlación de Pearson & $-.501 * *$ & $-.5 .22 * *$ \\
\hline & & Sig. (bilateral) & .000 & .000 \\
\hline
\end{tabular}


Se analizaron asimismo las correlaciones bilaterales entre la depresión y los indicadores de la CVRS. Para ello, se segmentaron los datos según las pacientes que reportaron progresión de enfermedad y las que no lo hicieron a fin de hacer un análisis más específico. Tal como se señala en la tabla anterior, la correlación entre la depresión y la salud general, evaluadas a través del GHQ-R $(p<0.01)$ y del SF-12 $(p<0.01)$, resultaron negativas, moderadas y significativas, lo que indica que una mayor presencia de depresión se asocia con un deterioro de la CVRS en las pacientes. Cuando se considera la progresión de la enfermedad, se aprecia la misma fuerza y dirección de la relación, siendo ambas significativas. En aquellas pacientes en que se identificó progresión de enfermedad se observó que la relación entre la depresión y la salud general de ambas medidas fue más fuerte, en comparación con aquellas que no la reportaron.

\section{DISCUSIÓN}

El presente estudio tuvo como propósito determinar las relaciones entre la CVRS, la depresión y la progresión de la enfermedad en una muestra de pacientes venezolanas sobrevivientes al cáncer de mama.

De acuerdo con los resultados hallados, las pacientes reportaron niveles bajos de depresión. La prevalencia de depresión grave, siguiendo los puntos de corte provistos por Rico et al. (2005), fue de $7.4 \%$. Este porcentaje es menor al hallado por Carvallo y Urbani (2007), quienes encontraron una prevalencia de $16.8 \%$ de depresión grave en pacientes oncológicos venezolanos. La prevalencia reportada en este estudio es cercana a los resultados de Granado y Trejo (2014), quienes reportaron $9 \%$ de depresión elevada en 55 pacientes con cáncer de mama venezolanas en la fase de diagnóstico. También es próximo al porcentaje hallado recientemente por Parra (2020) en pacientes con cáncer de mama venezolanas que se encontraban en las fases de tratamiento y libre enfermedad, que fue de 6,3\%. Curiosamente, estos últimos datos no difieren de la estimación de depresión grave anual reportada por la Organización
Panamericana de la Salud - OPS (2020) en Latinoamérica, la cual es que es de $6,7 \%$.

Los resultados parecen indicar que la sintomatología depresiva reportada en las pacientes es sugestiva de reacciones emocionales normales ante el proceso de la enfermedad. Si bien el cáncer genera un fuerte impacto emocional, es común que las personas diagnosticadas manifiesten síntomas depresivos, como una forma de distrés psicológico esperado ante la situación (Cruzado, 2014; Jaras y Gutiérrez, 2016; Holland et al., 2015). Los resultados hallados por Granado y Trejo (2014) y Parra (2020), así como los reportados en este trabajo permiten afirmar que, indistintamente de la fase de la enfermedad en la que se encuentren las pacientes venezolanas con cáncer de mama, la depresión elevada suele ser reducida.

Se ha evidenciado que los síntomas depresivos pueden fluctuar a lo largo de enfermedad, aunque tienen una mayor prevalencia en el primer año tras el diagnóstico de cáncer, y una tendencia a disminuir debido a los ajustes psicológicos que efectúan las pacientes, pero que también pueden reaparecer y aumentar ante la recurrencia y ya en la fase paliativa de la enfermedad (Bortolato et al., 2017; Cardoso et al., 2015). Algunos estudios (Grabsch et al., 2006; Lidgren et al., 2007) han hallado que las mujeres con cáncer de mama en estadios III y IV están en un mayor riesgo de sufrir depresión. Valderrama y Sánchez (2018) encontraron recientemente en su estudio que $25 \%$ de las pacientes colombianas con cáncer de mama en un estadio avanzado exhiben niveles de depresión grave. Así, es esencial la fase en que se halla la enfermedad para que se manifiesten e intensifiquen los síntomas depresivos en los pacientes oncológicos.

La CVRS de las pacientes, que fue evaluada mediante los dos instrumentos de medida relativos a este constructo, se ubicó entre niveles moderados y altos en ambas mediciones, siendo los puntajes semejantes y consistentes. Específicamente, cuando se examinaron los resultados del cuestionario SF-12, la salud física se halló más conservada que la salud mental. Para efectos de tales resultados, las pacientes mostraron una mejor salud luego de haber trascurrido el proceso de tratamiento, hallazgo similar al de Bardwell et al. (2003), quienes 
reportaron que, luego de tratamiento, una mejor CVRS se asociaba con una menor sintomatología física y con un aumento de la calidad del sueño en pacientes con cáncer de mama, resultados que contrastan con los de otros estudios (De Ligt et al., 2019; Lidgren et al., 2007; Schou et al., 2005), en los que se ha reportado una disminución de la CVRS como producto de los síntomas asociados a la enfermedad, los efectos secundarios de los tratamientos y las secuelas de las intervenciones quirúrgicas.

La muestra en esta investigación reportó en su mayoría haber manejado adecuadamente los efectos secundarios de los tratamientos, lo que sugiere una correcta evaluación de los mismos y un tipo de afrontamiento eficaz. Además, se evidenció que las pacientes tenían una edad media de 56 años y tres con la enfermedad, activas laboralmente y ejerciendo sus roles como madres, lo cual podría influir en la valoración positiva de su salud y lograran en consecuencia mantenerla. Por ejemplo, hay estudios que han reportado que las mujeres más jóvenes, laboralmente activas, con severas condiciones económicas y con un escaso apoyo social tienden a disminuir su CVRS (Cardoso et al., 2016; Fernández et al., 2020; Høyer et al., Lidgren et al., 2007; Rautalin et al., 2017). Aunque en este trabajo las pacientes sobrevivientes de cáncer de mama reportaron pertenecer en su mayoría a un estrato socioeconómico bajo, percibían tener apoyo social y familiar y llevaban a cabo activamente sus tareas diarias, lo que posiblemente había contribuido a amortiguar el estrés psicológico que podrían experimentar.

La depresión y la CVRS no estuvieron asociadas a la progresión de la enfermedad, evidenciándose la independencia entre estas variables. Sin embargo, al examinar la relación entre la depresión y la CVRS en la muestra total resultó ser negativa, moderada y significativa. La misma correlación se halló cuando se le evaluó en las pacientes que revelaron en su TC progresión de enfermedad y en las que esa progresión fue negativa, siendo en las primeras más sólida esa relación. En tal sentido, la depresión se asoció con un deterioro de la salud en las pacientes en general, indistintamente que hubiesen manifestado o no una progresión.
La fuerza de la relación reportada en aquellas pacientes que progresaron en la enfermedad refleja el riesgo mayor que tenían de que su salud se deteriorase al haber comorbilidad con depresión. Como se señaló anteriormente, es posible que ante la recurrencia del cáncer los pacientes puedan reportar niveles de depresión severa o clínica (Bortolato et al., 2017).

Lo anterior es especialmente importante para el abordaje psicológico temprano en aquellos casos que sean clínicos, pues la depresión puede llegar a cronificarse en algunas personas y producir un elevado nivel de insatisfacción y una valoración de incapacidad e ineficacia ante todas las situaciones de la vida, al punto de que desarrollen predisposiciones personales, relativamente estables, a experimentar estados depresivos frecuentes o la depresión como un rasgo de la personalidad (Beck, 1987; Carbonell, Grau y Grau, 2003) que no fue evaluado en la muestra de estudio. Las pacientes con antecedentes o predisposiciones a la depresión patológica ante el diagnóstico de cáncer tienen una mayor tendencia a sufrir episodios depresivos graves y recurrentes que inciden en su ajuste a la enfermedad y a su pronóstico (Bernal y Muñoz, 2016).

El hecho de que no se encontrara una asociación entre la depresión y la progresión de enfermedad guarda correspondencia con los resultados del estudio de Mansano y Ceolim (2017) con una muestra de pacientes con cáncer de mama colombianas, en el que no hallaron evidencia sobre la relación causal entre la depresión y la progresión de la enfermedad oncológica, evaluada con los resultados de las pruebas del receptor HER-2 y de los receptores hormonales de estrógeno y progesterona. Aunque existe cierta evidencia sobre la relación entre la depresión y la progresión de la enfermedad oncológica (Pinquart y Duberstein, 2010; Spiegel y Giese-Davis, 2003), los resultados del estudio antes citado, al igual que los presentados aquí, dan apoyo a lo afirmado por Lutgendorf y Andersen (2015) de que los hallazgos sobre tal vínculo aún no son concluyentes. Sin embargo, no se descarta que la naturaleza del estudio, el tipo de muestreo, las características de la muestra y el uso de un solo método para evaluar la progresión hayan influido 
en los resultados obtenidos. Incluso así, sí logra proveer más evidencia sobre la relación entre la depresión y la CVRS en las pacientes con cáncer de mama (Cardoso et al., 2016; Lidgren et al., 2007; Moseholm et al., 2016). Los estudios antes citados han reportado que la depresión tiene efectos negativos en el funcionamiento físico y social, en el desempeño de los roles y el estado emocional en general y que tienden agudizarse más cuando existe una evaluación negativa de la salud y la percepción de bajo apoyo social.

Lutgendorf y Andersen (2015), han explicado que muchos factores psicosociales perjudiciales para los individuos -tales como las dificultades económicas, el bajo apoyo social e interpersonal, la depresión, etc.-, que al interactuar con otros factores del medio ambiente, producen estrés, y que al cronificarse, activan las vías neuroendocrinas y autonómicas y el eje hipotalámico pituitario adrenal (HPA). La depresión incide en la regulación del HPA, que afecta negativamente al sistema inmune y los procesos inflamatorios y angiogenéticos, que se encuentran vinculados con el control y el desarrollo de la progresión del cáncer. Aunque la depresión no se asoció con la progresión de enfermedad, se comprende que puede tener efectos sobre la salud que, al deteriorarla, incrementa el riesgo de progresión y mortalidad por cáncer. Precisamente, la depresión puede producir baja adherencia al tratamiento e incluso su abandono, lo que aumentaría la probabilidad de que el cáncer avance (Reiche et al., 2004: Spiegel y Giese-Davis, 2003).

Especial importancia tiene el adecuado apoyo social percibido en las pacientes en este estadio, el cual parece ser un factor de protección contra la depresión. Los estudios han encontrado que una elevada depresión ha estado estrechamente asociada con un bajo apoyo social percibido en pacientes con cáncer de mama (Lidgren et al., 2007; Moseholm et al., 2016).

Pinquart y Duberstein (2010), en un metaanálisis sobre depresión y mortalidad por cáncer, reportaron que los pacientes oncológicos que contaban con una amplia red social y percibían un buen apoyo de la misma tenían un menor riesgo de morir por esta enfermedad. Además, se puede inferir que las pacientes poseen otras fortalezas personales que las ayudan a mantener su calidad de vida y su salud. Se ha reportado que aspectos como el afrontamiento, optimismo, esperanza, resiliencia, espiritualidad, optimismo y apoyo social mejoran la salud de las pacientes con cáncer de mama (Franco et al., 2019; Juárez y Landero, 2009; Lutgendorf y Andersen,2015) y pueden actuar como amortiguadores ante el estrés y así evitar su progresión. Futuros estudios podrían examinar el rol mediador o moderador de estas variables en la relación entre depresión y CVRS, específicamente en el contexto venezolano.

Este trabajo permite develar que las pacientes con cáncer de mama venezolanas son diagnosticadas, en promedio, en edades en las que se encuentran activas en sus diferentes roles de la vida diaria, con una sobrevivencia aproximada de tres años, madres, de nivel socioeconómico bajo, activas laboralmente y por lo regular casadas o solteras. Además, siguiendo los resultados reportados, las pacientes denotan en general un buen ajuste psicológico, manifestado en niveles de CVRS conservados y una menor incidencia de depresión y de progresión de enfermedad, que pueden estar influenciados por la evaluación que ellas hacen sobre la enfermedad, el afrontamiento, el apoyo social percibido y otras características individuales. Los resultados de este estudio representan otra aportación a lo que se ha reportado sobre la incidencia de los factores emocionales en los procesos de salud-enfermedad de las pacientes venezolanas con cáncer de mama (Altuve, 2020; Parra, 2020), una de las neoplasias más diagnosticadas en el país. No obstante, es necesario seguir profundizando en los hallazgos a través de estudios longitudinales que hagan posible valorar la depresión en diferentes momentos, específicamente en las pacientes que progresan en la enfermedad, lo que podría ser relevante para corroborar la asociación entre la depresión y dicha progresión. Es necesario también que los estudios en el área puedan ampliarse a muestras de pacientes con otro tipo de neoplasias.

Algunas limitaciones de este estudio fueron, en primer lugar, no contar con estudios psicométricos más apropiados de los cuestionarios HADS y GHQ-R. El HADS es utilizado con cierta frecuencia para valorar la depresión en los pacientes oncológicos por la bondad que posee de no contener 
reactivos referentes a ciertos síntomas somáticos que se manifiestan en los estados depresivos. Aunque se reportó una adecuada consistencia interna en el estudio piloto efectuado en esta investigación, es importante que se puedan llevar a cabo en un futuro estudios psicométricos más amplios y más estructurados sobre esta escala, así como también del GHQ-R, empleando para ello más participantes con diferentes tipos de cáncer, de modo que se puedan disponer de puntos de cortes locales que permitan detectar la depresión severa y la disminución significativa de la salud mental en general de los pacientes oncológicos venezolanos. Por otro lado, no fue posible disponer de otras evaluaciones de la progresión de enfermedad aparte de la tomografía computarizada, tales como la prueba del receptor HER-2 o la de los receptores hormonales de estrógeno y progesterona, que son muy útiles para evaluar la progresión del cáncer de mama, debido a que no fueron proporcionadas por todas las pacientes de la muestra.

La relevancia de estos resultados se orienta al diseño de programas psicoeducativos e interven- ciones de asesoramiento psicológico para promover la concienciación en la prevención y detección temprana del cáncer, dada su incidencia en mujeres de edades en las que aún se encuentran activas.

La mayoría de las pacientes reportaron no asistir a recibir apoyo psicológico, lo que parece indicar que aún se mantiene la desestimación del rol del psicólogo en las unidades de oncología, tal como lo encontró Oroza (1999) en un estudio hecho en el país. De la mano con esto, Cardoso et al. (2015) confirmaron que la depresión en las pacientes con cáncer de mama se asociaba con no contar con el apoyo de algún profesional de la salud mental. En tal sentido, es importante seguir comprendiendo que "el acompañamiento psicológico forma parte de todos los tratamientos que recibe el paciente oncológico" (Altuve, 2017, p. 3), e instar a que la brecha entre psicólogos y oncólogos se haga cada vez más estrecha, de modo que se estimule el trabajo en equipo para así promover un abordaje integral que favorezca la salud y calidad de vida de todos los pacientes con diagnóstico de cáncer.

Citación: Altuve B., J.J. y Parra, A.A. (2022). Calidad de vida relacionada con la salud, depresión y progresión de enfermedad en sobrevivientes de cáncer de mama venezolanas. Psicología y Salud, 32(1), 137-149. https:// Doi.org/10.25009/pys.v32il.2720.

\section{REFERENCIAS}

Altuve, A. (2014). Entre la prevención y la angustia. Reportaje interpretativo sobre la crisis de salud y el control del cáncer en Venezuela. Trabajo de Grado. Caracas: Universidad Central de Venezuela.

Altuve, J. (2017). La psicooncología: aspectos generales (Manuscrito no publicado). Caracas: Servicio Oncológico Hospitalario del Instituto Venezolano de los Seguros Sociales.

Altuve, J. (2020). Distrés psicológico, miedo a la recurrencia del cáncer y calidad de vida relacionada con la salud en pacientes sobrevivientes de cáncer de mama. Psicooncología, 17(2), 239-253.

Ashing-Giwa, K., Rosales, M., Lai, L. y Weitzel, J. (2012). Depressive symptomatology among Latin breast cancer survivors. Psycho-Oncology, 22(4), 845-853.

Bardwell, W., Major, J., Rock, C., Newman, V., Thomson, C., Chilton, J., Dimsdale, J. y Pierce, P. (2003). Health-related quality of life in women previously treated for early-stage breast cancer. Psycho-Oncology, 5(13), 595-604.

Beatty, L. y Kisanne, D. (2017). Anxiety and depression in women with breast cancer. Cancer Forum, 41(1), 55-61.

Beck, A. (1987). Terapia cognitiva de la depresión. Bilbao: Desclée de Brouwer.

Bernal, G. y Muñoz, A. (2016). Tratamiento farmacológico de la depresión en cáncer. Psicooncología, 13(2-3), 249-270.

Bortolato, B., Nyphantis, T., Vapione, S., Perini, G., Maes, M., Morris, G., Kubera, M., Kohler, C., Fernandes, B., Stubbs, B., Pavilidis, N. y Carvalho, A. (2017). Depression in cancer: the many biobehavioral pathways driving tumor progression. Cancer Treatment Reviews, 52, 58-70.

Calderon, C., Carmona B., A., Hernandez, R., Ghanem, I., Castelo, B., De Castro, E., Ferreira, E., Ciria, L., Muñiz, M. y Jimenez F., P. (2019). Effects of pessimism, depression, fatiga, and pain on functional health-related quality of life in patients with resected non-advanced breast cancer. The Breast, 44, 108-112. 
Carbonell, M., Grau, R. y Grau, J. (2003). El Inventario de Depresión Estado-Rasgo (IDERE): desarrollo de una versión cubana. Terapia Psicológica, 21(2), 121-135.

Cardoso, G., Graca, J., Klut, C., Trancas, B. y Papolia, A. (2015). Depression and anxiety symptoms following cancer diagnosis: a cross-sectional study. Psychology, Health \& Medicine, 21(5), 1-14.

Caruso, R., Nanni, M., Riba, M., Sabato, S., Mitchell, A., Croce, E. y Grassi, L. (2016). Depressive spectrum disorders in cancer: prevalence, risk factor san screening for depression: a critical review. Acta Oncológica, 56(2), 146-155.

Carvallo, M. y Urbani, S. (2007). Expreriencia de la Unidad de Psicoonologia del Servicio de Radioterapia y Medicina Nuclear. Revista Venezolana de Oncología, 19(2), 1-2.

Costanzo, E., Sood, A. y Lutgendorf, S. (2011). Biobehavioral influences on cancer progression. Immunology and Allergy Clinics, 31(1), 109-132.

Cruzado, J. (2014). Manual de Psicooncología. Tratamientos psicológicos para el paciente con cáncer. Madrid: Pirámide.

De Ligt, K., Heins, M., Verloop, J., Ezendam, N., Smorenburg, C., Korevaar, J. y Siesling, S. (2019). The impact of health symptoms on health-related quality of life in early-stage breast cancer survivors. Breast Cancer Research and Treatment, 178(3), 703-711.

Die Trill, M. (2003). Psico-Oncología. Madrid: ADES.

Diz, R., Garza, C., A., Olivas, V., E.K., Montes, E., J.G., Fernández, L. y G.S. (2019). Cáncer y depresión: una revisión. Psicología y Salud, 29(1), 115-124.

Fernández, N., Pérez, B., Guerrero, A., Casas, A., Bermejo, B., Baena, J., Antolín, S., Sánchez R., P., Ramos, M., García S., J., Antón, A., Muñoz, M., Juan, A., Jara, C., Chacón, J., Arcusa, A., Gil, M., Adrover, E., Oltra, A., Brunet, J., González, S. y Pollán, M. (2020). Primary breast cancer and health related quality of life in Spanish women: The EpiGEICAM case-control study. Scientific Reports, 7741(10), 1-13.

Franco, S., Zuluaga, M.A., Vinaccia, S., Raleigh, R. y Martínez, G. (2019). Variables salutogénicas y patogénicas, imagen corporal y calidad de vida relacionada con la salud en pacientes con cáncer de mama. Psicología y Salud, 29(2), 225-235.

Goldberg, D.P. y Hillier, V.F. (1979). A scaled version of the General Health Questionnaire. Psychological Medicine, 9, 139-145.

Grabsch, B., Clarke, D., Love, A., McKenzie, D., Snyder, R., Bloch, S., Smith, G. y Kissane, D. (2006) Psychological morbidity and quality of life in women with advanced breast cancer: a cross-sectional survey. Paliative Support Care, 4(1), 47-56.

Granado, B. y Trejo, E. (2014). Medición de los trastornos adaptativos en mujeres con cáncer de mama (Trabajo de Grado). Caracas: Universidad Central de Venezuela.

Hernández, N., Salas, A. y Altuve, J. (2020). Afrontamiento, funcionamiento familiar y calidad de vida relacionada con la salud en cuidadores venezolanos de pacientes con cáncer. Psicología y Salud, 30(2), 161-172.

Hernández, R., Fernández, C. y Baptista, L. (2012). Metodología de la investigación. México: McGraw-Hill.

Holden, A., Ramirez, A. y Gallion, K. (2014). Depressive symptoms in Latin breast cancer survivors: A barrier to cancer screening. Health Psychology, 33(4), 242-248.

Holland, J., Breitbart, W., Butow, P., Jacobsen, P., Loscalzo, M. y McCorkle, R. (2015). Psycho-Oncology (3th ed.). New York: Oxford University Press.

Høyer, M., Johansson, B., Nordin, K., Bergkvist, L., Ahlgren, J., Lidin-Lindqvist, A., Lambe, M. y Lampic, C. (2011). Health-related quality of life among women with breast cancer: A population-based study. Acta Oncologica, 50(7), 1015-1026.

Jaras, C. y Gutiérrez, Y. (2016). Psicooncología. Enfoques, avances e investigaciones. Santiago de Chile: Nueva Mirada.

Juárez, D. y Landero, R. (2009). Variables psicosociales y salud en mujeres con cancer de mama. Summa Psicologica, 6(2), 79-88.

Kerlinger, F. y Lee, H. (2002). Investigación del comportamiento. Buenos Aires: McGraw-Hill.

Lee, M., Martinez, D., Gonzalez, B., Small, B., Lechner, S., Antoni, M., Vinard, A., Krause, M., Meade, C. y Jacobsen, P. (2017). Anxiety ans depression en Spanish-speaking Latin cancer patients prior to strarting chemotherapy. Psycho-Oncology, 27(1), 333-338.

Lidgren, M., Wilking, N., Jönsson, B. y Rehnberg, C. (2007). Health related quality of life in different states of breast cancer. Quality of Life Research, 16(6), 1073-1081.

Linden, W., Vodermaier, A., MacKenzie, R. y Greig, D. (2012). Anxiety and depression after cancer diagnosis: prevalence rates by cancer type, gender, and age. Journal of Affective Disorders, 141(2), 434-351.

Lutgendorf, S. y Andersen, B. (2015). Biobehavioral approaches to cancer progression and survival. American Psychologist, 70(2), 186-197.

Lutgendorf, S. y Sood, A. (2011). Biobehavioral factors and cancer progression: Physiological pathways and mechanisms. Psychosomatic Medicine, 71, 724-730.

Mansano, T. y Ceolim, M. (2017). Asociación de pronóstico clínico desfavorable y duración del sueño en pacientes con cáncer de mama. Revista Latinoamericana Enfermagem, 25, 1-9. 
Massie, M. (2004). Prevalence of depression in patients with cancer. Journal of the National Cancer Institute Monographs, 32, $57-71$.

Moseholm, E., Rydahi-Hansen, S., Overgaard, D., Wengel, H., Frederiksen, R., Brandt, M. y Lindhardt, B. (2016). Health-realted quality of life, anxiety and depression in the diagnostic phase of suspected cancer, and the influence of diagnosis. Health and Quality of Life Outcomes, 14(1), 80.

Organización Panamericana de la Salud (2020). La salud mental en la región de las Américas. Washington: OpS.

Oroza, R. (1999). El psicólogo en un servicio de oncología. Extramuros, 11, 225-245.

Park, E., Gelber, S., Rosenberg, S., Seah, D., Schapira, L., Come, S. y Partridge, A. (2018). Anxiety and depression in young women with metastatic breast cancer: a cross-sectional study. Psychosomatics, 59(3), 251-258.

Parra, A. (2020). Depresión en pacientes con cáncer de mama (Trabajo inédito de Especialidad). Caracas: Universidad Central de Venezuela.

Pilevarzadeh, M., Amirshahi, M., Afsargharehbagh, R., Rafiemanesh, H., Hashemi, S.M. y Balouchi, A. (2019). Global prevalence of depression among breast cancer patients: a systematic review and meta-analysis. Breast Cancer Research and Treatment, 176, 1-15.

Pinquart, M. y Duberstein, P. (2010). Depression and cancer mortality: A meta-analysis. Psychological Medicine, 40, 1797-1810.

Rautalin, M., Färkkila, N., Sintonten, H., Saato, T., Taari, K., Jahkola, T. y Risto, R. (2017). Health-related quality of life in different states of breast cancer-comparing different instruments. Acta Oncologica, 57(5), 622-628.

Reich, M., Lesur, A. y Perdrizet-Chevallier, C. (2008). Depression, quality of life and breast cancer: a review of the literature. Breast Cancer Research and Treatment, 110(1), 9-17.

Reiche, E., Nunes, S. y Morimoto, H. (2004). Stress, depression, the immune system, and cancer. Lancet Oncology, 5, 617-625.

Rico, J., Restrepo, M. y Molina, M. (2005). Adaptación y validación de la escala hospitalaria de ansiedad y depresión (HADS) en una muestra de pacientes con cáncer del Instituto Nacional de Cancerología de Colombia. Revista del Laboratorio de Psicometría, 3, 73-86.

Rodríguez, M., Lacruz, J., Márquez, M., López, F., Borges, R. y Delgado, J. (2014). Factores pronósticos y predictivos en el cáncer de mama con sobre expresión del HER-2/Neu. Revista Venezolana de Oncología, 26(3), 175-186.

Rutherford, C. (2018). Health-related quality of life in cancer. En I. Oliver (Ed.): The MASCC textbook of cancer supportive care and survivorship (pp. 109-125). Sidney: Springer.

Schou, I., Ekeberg, Ø., Sandvik, L., Hjermstad, M. y Ruland, C. (2005). Multiple predictors of health-related quality of life in early stage breast cancer. Data from a year follow-up study compared with the general population. Quality of life Research, 14(8), 1813-1823.

Smith, H. (2015). Depression in cancer patiens: Pathogenesis, implications and treatment (Review). Oncology Letters, 9, 1509-1514.

Spiegel, D. y Giese-Davis, J. (2003). Depression and cancer: mechanisms and disease progression. Biological Psychiatry, 54(3), 269-282.

Valderrama, M. y Sánchez, R. (2018). Trastornos de ansiedad y depresión en la relación con la calidad de vida de pacientes con cáncer de mama en estadio localmente avanzado o diseminado. Revista Colombiana de Psiquiatría, 47(4), 211-220.

Villa, G., Zuluaga, C. y Restrepo, L. (2013). Propiedades psicométricas del Cuestionario de Salud General de Goldberg GHQ-12 en una institución hospitalaria de la ciudad de Medellín. Avances en Psicología Latinoamericana, 31(3), 532-545.

Ware, J.E., Jr., Kosinski, M. y Keller, S.D. (1996). A 12-item short-form health survey: construction of scales and preliminary tests of reliability and validity. Medical Care, 34, 220-233.

Yfantis, A., Intas, G., Tolia, M., Nikolaou, M., Tsoukalas, N., Lymperi, M., Kyrgias, G., Zografos, G. y Kontos, M. (2018). Health-related quality of life of young women with breast cancer. Review of the literature. Journal of the Balkan Union of Oncology, 23(1), 1-6.

Young, K. y Singh, G. (2018). Biological mechanisms of cancer-induced depression. Fontiers in Psychiatry, 9, 299-308.

Zabora, J., Brintzenhofeszoc, K., Curbow, B., Hooker, C. y Piantadosi, S. (2001). The prevalence of psychological distress by cancer site. Psycho-Oncology, 10(1), 19-22.

Zigmond, A.S. y Snaith, R.P. (1983). The Hospital Anxiety and Depression Scale. Acta Psychiatrica Scandinavica, 67, 361-370.

Instituto de Investigaciones Psicológicas - Universidad Veracruzana ISSN impreso: 1405-1109
Psicología y Salud, Vol. 32, Núm. 1: 137-149, enero-junio de 2022 https://doi.org/10.25009/pys.v32i1.2720 